\title{
Is chronic renal failure a risk factor for the development of erosive osteoarthritis?
}

\author{
I J S DUNCAN, ${ }^{1} \mathrm{~N}$ P HURST, ${ }^{1}$ A DISNEY ${ }^{2}$ R SEBBEN ${ }^{3}$ AND S C MILAZZO \\ From the ${ }^{1}$ Rheumatology Unit, the ${ }^{2}$ Renal Unit, and the ${ }^{3}$ Department of Radiology, The Queen Elizabeth \\ Hospital, Woodville, South Australia 5011
}

SUMMARY Erosive osteoarthritis of the hands of unusually early onset and severity was seen in two patients treated for chronic renal failure by long term haemodialysis and renal homograft respectively. The significance of this observation is discussed in the light of previous studies of erosive arthropathy in patients with chronic renal failure. Factors associated with chronic renal failure may predispose to the development of erosive osteoarthritis.

Key words: haemodialysis, hyperparathyroidism.

During a recent survey of patients receiving long term haemodialysis in our hospital we noted that erosive osteoarthritis (OA) was relatively frequent, often severe, and in some patients developed at a relatively young age. ${ }^{1}$ We had also seen similarly severe erosive OA develop several years after successful renal transplantation. Although erosive arthropathy of the hands has been described previously in patients with chronic renal failure, it has not been reported in patients who have had successful renal homografts. Furthermore, there is some disagreement in published work about the morphology of the erosive arthropathy occurring in chronic renal failure and whether or not it is associated with significant joint destruction. Here we describe two patients with erosive arthropathy resembling erosive $\mathrm{OA}$ and discuss whether chronic renal failure constitutes a risk factor for the development of this condition.

\section{Case histories}

CASE 1

Case 1 was a male clerk, who developed chronic renal failure secondary to focal glomerulonephritis in 1969 at age 22 and underwent bilateral nephrectomy. Two renal grafts (1970 and 1971) lasted only weeks, and the patient was subsequently treated

Accepted for publication 14 July 1988.

Correspondence to Dr N P Hurst, Rheumatology Unit, The Queen Elizabeth Hospital, Woodville, South Australia 5011. with long term haemodialysis. Despite parathyroidectomy in 1975 recent bone biopsy showed active hyperparathyroidism. He also had secondary iron overload with a serum ferritin of $2140 \mu \mathrm{g} / \mathrm{l}$ (normal $<250 \mu \mathrm{g} / \mathrm{l}$ ). There was no family history of OA. In 1978 at age 31 he developed intermittent pain and swelling in the distal interphalangeal (DIP) joints of the fingers and interphalangeal (IP) joints of each thumb. These episodes became less frequent but left minor functional loss in both hands. In 1986 he also developed bilateral carpal tunnel syndrome owing to $\beta_{2}$ microglobulin related amyloidosis. On clinical examination he had Heberden's nodes and DIP joint deformities typical of erosive OA. Serial radiographs showed progressive erosive $\mathrm{OA}$ and subperiostial resorption of the radial aspect of the middle phalanges typical of hyperparathyroidism (Fig. 1).

\section{CASE 2}

Case 2 was a male clerk, who developed chronic renal failure secondary to chronic glomerulonephritis in 1962 at age 27 . He underwent haemodialysis for three years and then received a renal graft, which has functioned successfully since 1966 (current creatinine $140 \mu \mathrm{mol} / \mathrm{l}$ ). Parathyroid hormone concentrations were normal. His maternal grandmother had nodal OA. His present treatment includes azathioprine (50 mg daily) and prednisolone ( $7.5 \mathrm{mg}$ daily), but previous high dose steroid treatment was associated with episodes of osteo- 
necrosis affecting the femoral condyles. In 1965 at age 30 soft tissue swelling and early joint space narrowing was apparent on $x$ ray examination. By 1977 the radiological changes were florid, and these have since progressed to involve the IP joints of all fingers and both thumbs. On examination there were florid changes of erosive $\mathrm{OA}$ with fixed flexion? deformities and marked reduction of flexion. He $\frac{}{\sigma}$

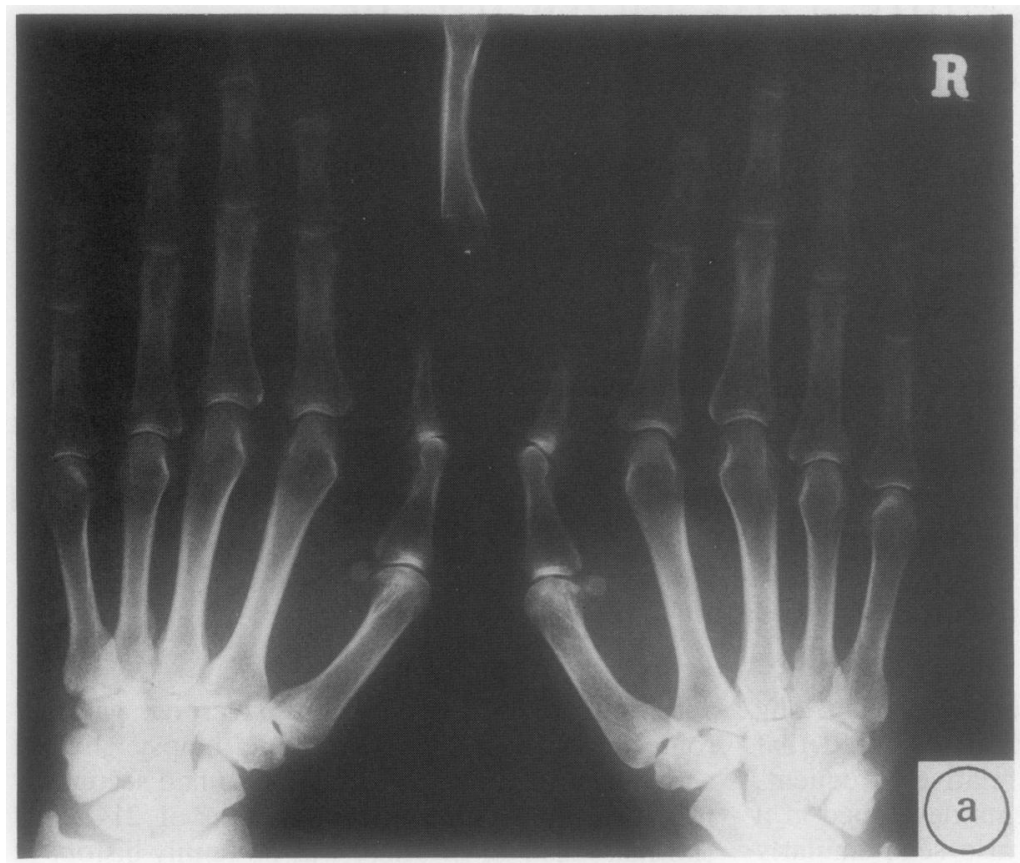

Fig. 1 Serial radiographs showing (a) early joint space narrowing and some erosive changes in the distal interphalangeal (DIP) joints in 1978 (age 31 years) and (b) progressive involvement of DIP joints of all fingers and interphalangeal joints of both thumbs by 1988.

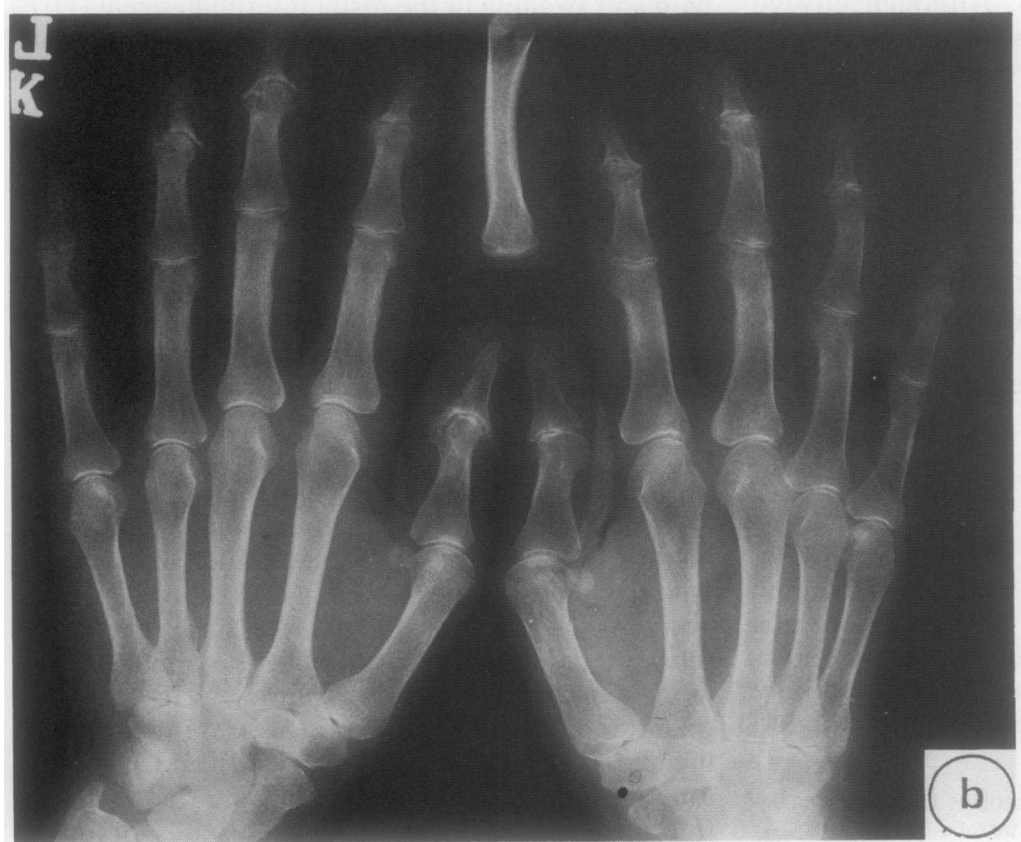




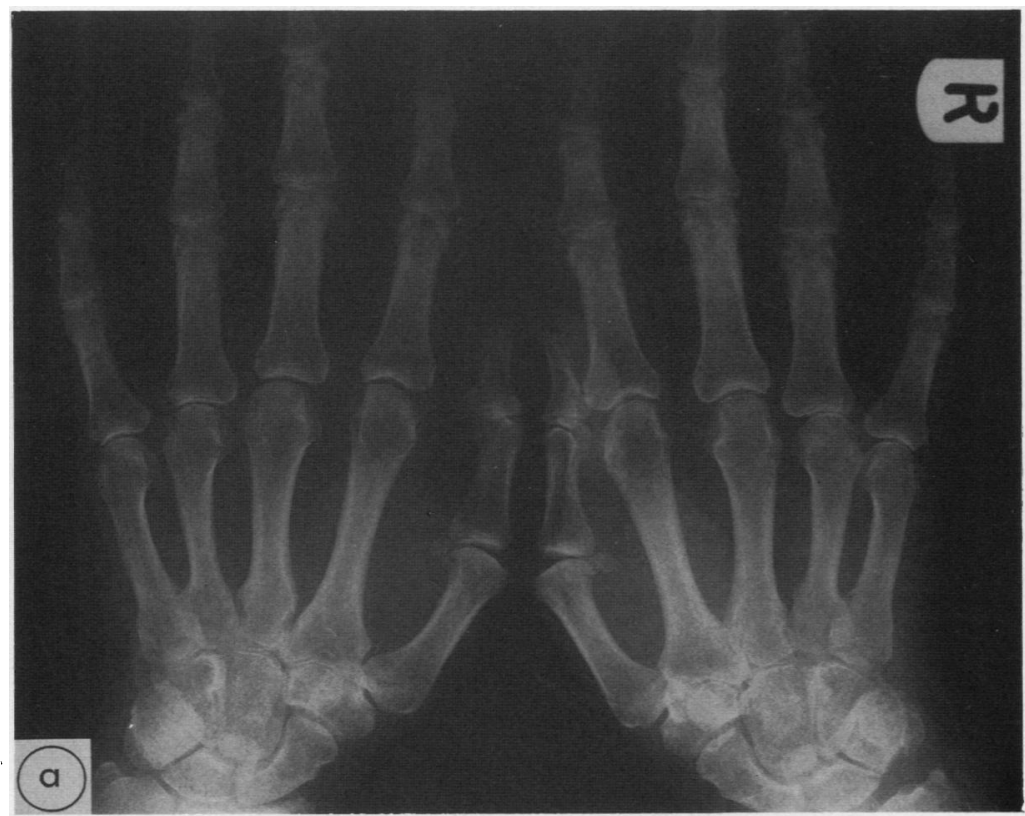

Fig. 2 Serial radiographs showing (a) early joint space narrowing, capsular calcification, and soft tissue swelling in 1965 (age 30 years), (b) progressing to severe destructive changes affecting mainly interphalangeal joints by 1977. Minor joint space narrowing is apparent in some metacarpophalangeal joints.

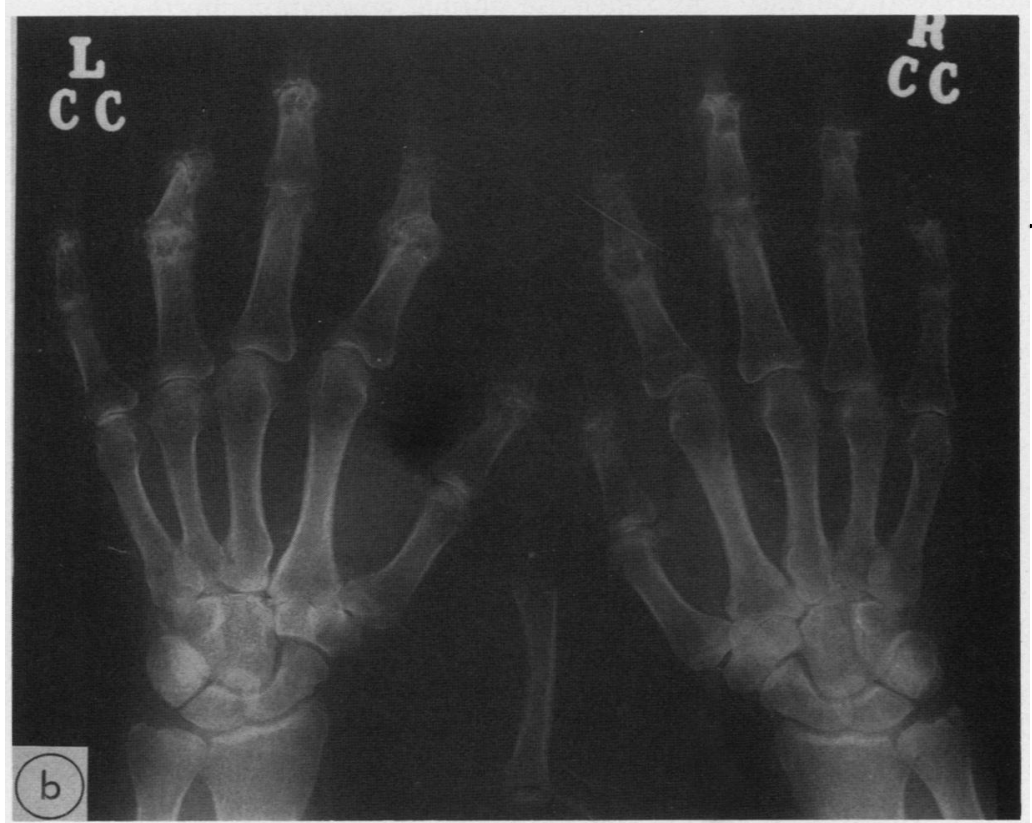

was unable to oppose his thumb and index finger. Serial radiographs showed severe destructive erosive OA in all DIP and proximal interphalangeal (PIP) joints (Fig. 2).

\section{Discussion}

A wide variety of musculoskeletal problems have been reported in patients with chronic renal failure. 
These include renal osteodystrophy, avascular necrosis of bone, crystal induced arthropathies, and $\beta_{2}$ microglobulin related amyloidosis of bones and joints. Erosive arthropathy of the hands has also been described; in some reports the appearances are of small juxta-articular erosions affecting metacarpophalangeal (MCP), PIP, and DIP joints without any significant joint destruction. ${ }^{2}$ Other reports describe an erosive and destructive arthropathy, affecting PIP, DIP, and first carpometacarpal (CMC) joints, which resembles erosive $\mathrm{OA}^{3}$ Whether these descriptions represent two separate entities or whether they have a similar aetiology remains unclear. Both the patients we report here developed, at a young age, a destructive erosive osteoarthropathy which except for the absence of involvement of the first CMC joints was indistinguishable clinically and radiologically from erosive OA. ${ }^{1}$ Although this condition has been described previously in patients receiving long term haemodialysis, it has not been described in patients who have undergone successful renal transplantation after only a relatively short period of haemodialysis.

A number of authors have described juxtaarticular erosions in the finger joints without associated degenerative change in patients with chronic renal disease,${ }^{34}$ and this has usually been attributed to secondary hyperparathyroidism. In contrast, others have described a destructive arthropathy resembling erosive $\mathrm{OA},{ }^{25}$ and there is disagreement as to whether this is a separate entity or part of the spectrum of hyperparathyroidism.

The first report in 1963, by Bywaters et al, of erosive arthropathy in patients with hyperthyroidism described subchondral erosions in wrists, MCP joints, and large peripheral joints. ${ }^{6}$ No comment was made about DIP or PIP joints, though a radiograph showing possible DIP joint erosions was included. Secondary degenerative changes were described in large and medium sized joints, but not in MCP joints, and were attributed to collapse of softened juxta-articular bone. Resnick later reported more widespread erosive changes in the hands of four patients with hyperparathyroidism, two of whom had chronic renal failure but had not received haemodialysis. ${ }^{3}$ Joint space narrowing was present in carpal joints and DIP joints, but he emphasised its absence in the MCP and PIP joints.

Subsequent reports have been conflicting. In a retrospective study Sundaram et al described erosive small joint changes in 24 of 80 patients undergoing long term haemodialysis. ${ }^{5}$ Juxta-articular erosions frequently affected the DIP and MCP joints but not the PIP joints. In some patients DIP joint erosions were associated with degenerative destructive changes resembling erosive $\mathrm{OA}$, but as the number of affected patients and their age were not specified this might have been an unconnected age relatedo़? process. Rubin et al, in a study of 59 patientso undergoing long term haemodialysis, showed $a$ 을 similar high prevalence of small joint erosions, but $t^{\frac{\rho}{5}}$ in contrast did not identify any associated degenera- $-\frac{\pi}{\Omega}$ tive changes. ${ }^{4}$

A recent radiological survey of 81 patients under- going long term haemodialysis identified 32 with. severe 'erosive osteoarthropathy' affecting almost $\vec{\omega}$ equally DIP, PIP, and first CMC joints. ${ }^{2}$ Erosions ofo the MCP joints were infrequent. Affected joints were often grossly narrowed with the 'gull's wing"' appearance and lateral subluxation of DIP joints $\omega$ typical of erosive OA. ${ }^{1}$ This arthropathy was clearly more severe than that described by other authorsw and showed the female preponderance typical of erosive OA. ${ }^{1}$ Surprisingly, the authors concluded. that the radiological changes were not characteristic of erosive OA as there was osteopenia, early cystic change, sometimes soft tissue calcification, and lack ${ }^{-}$ of osteophytes. Although the calcification is noteo typical, the radiological joint changes illustrated in their article would be hard, if not impossible, to distinguish from the erosive OA seen in an elderly but otherwise normal population. Furthermore, it has been suggested that osteopenia may predisposea to this form of osteoarthritis. ${ }^{7}$

During a recent survey of 95 patients receiving long term haemodialysis in our hospital it was noted that 59 had small joint symptoms, of whom 14 had erosive OA. ${ }^{8}$ Erosive $\mathrm{OA}$ increased in prevalence with age, was more common in women, and almost exclusively affected the DIP, PIP, and first CMC joints. Two patients, one of whom is presented here were under 40 years of age at the time of onset. InD. contrast, the prevalence of small joint erosions typical of hyperparathyroidism, affecting MCP or IP joints and unassociated with degenerative change 0 increased with duration of haemodialysis rather than with age and affected MCP as well as Ifo joints.

In addition to hyperparathyroidism, other endo crine or metabolic factors may have a role in the development of premature erosive $\mathrm{OA}$ in patientsu with renal disease. One patient described by Naidich et al developed severe secondary iron overloa associated with $\mathrm{Fe}_{3} \mathrm{O}_{4}$ deposition in the soft tissues? of the fingers. ${ }^{2}$ Despite chelation treatment he्? developed extensive chondrocalcinosis and a de $=0$ forming erosive OA of IP joints and wrists. We have not found any association among our patients receiving long term haemodialysis between develop $\frac{\rho}{\mathbb{Q}}$ ment of small or large joint osteoarthritis and raise ${ }^{\mathbb{R}}$
serum ferritin concentrations.

In conclusion, the two cases presented here 
strongly suggest that factors associated with chronic renal failure predispose to the early development of erosive OA. Successful transplantation did not appear to have mitigated against progression of erosive $\mathrm{OA}$ in case 2 . It is possible that osteopenia, related either to corticosteroid treatment or to other effects of renal functional impairment, may be a significant contributory factor. To what extent hyperparathyroidism contributes to this problem is uncertain as the distribution of joint involvement in the two conditions is different. If it is contributory other, perhaps mechanical, factors may determine which joints develop frank degenerative change. This would be consistent with the hypothesis proposed by Bywaters et al that collapse of softened juxta-articular bone is one mechanism of joint damage in such patients. ${ }^{6}$

To investigate further the nature and extent of the hand arthropathy in these patients we are currently undertaking a survey of all patients in our hospital receiving haemodialysis and those with a renal transplant.

\section{References}

1 Ehrlich G E. Erosive inflammatory and primary generalised osteoarthritis. In: Moskowitz R W, Howell D S, Goldberg V M, Mankin H J, eds. Osteoarthritis-diagnosis and management. Philadelphia: Saunders, 1984: 201-4.

2 Naidich J B, Karmel M I, Mossey R T, Bluestone P A, Stein $H$ I. Osteoarthropathy of the hand and wrist in patients undergoing long term haemodialysis. Radiology 1987; 164: 205-9.

3 Resnick D L. Erosive arthritis of the hand and wrist in hyperthyroidism. Radiology 1974; 110: 263-9.

4 Rubin L A, Fam A G, Rubenstein J, Cambell J, Saiphoo C. Erosive azotaemic osteoarthropathy. Arthritis Rheum 1984; 27: 1086-94.

5 Sundaram M, Wolverson M K, Heiberg E, Grider R D. Erosive azotemic osteodystrophy. AJR 1981; 136: 363-7.

6 Bywaters E G L, Dixon A St J, Scott J T. Joint lesions of hyperparathyroidism. Ann Rheum Dis 1963; 22: 171-87.

7 Smythe H A. Osteoarthritis, insulin and bone density. $J$ Rheumatol 1987; 14 (suppl 14): 91-3.

8 Hurst N P, van den Berg R, Disney A. et al. Dialysis related arthropathy: a survey of 95 patients undergoing chronic haemodialysis with special reference to $\beta_{2}$-microglobulin related amyloidosis. Ann Rheum Dis (in press). 